\title{
Tc1-like transposable elements in plant genomes
}

\author{
Yuan Liu ${ }^{1,2}$ and Guojun Yang ${ }^{1,2^{*}}$
}

\begin{abstract}
Background: The Tc1/mariner superfamily of transposable elements (TEs) is widespread in animal genomes. Mariner-like elements, which bear a DDD triad catalytic motif, have been identified in a wide range of flowering plant species. However, as the founding member of the superfamily, Tc1-like elements that bear a DD34E triad catalytic motif are only known to unikonts (animals, fungi, and Entamoeba).

Results: Here we report the identification of TC1-like elements (TLEs) in plant genomes. These elements bear the four terminal nucleotides and the characteristic DD34E triad motif of TC1 element. The two TLE families (PpTC1, PpTc2) identified in the moss (Physcomitrella patens) genome contain highly similar copies. Multiple copies of PpTc1 are actively transcribed and the transcripts encode intact full length transposase coding sequences. TLEs are also found in angiosperm genome sequence databases of rice (Oryza sativa), dwarf birch (Betula nana), cabbage (Brassica rapa), hemp (Cannabis sativa), barley (Hordium valgare), lettuce (Lactuta sativa), poplar (Populus trichocarpa), pear (Pyrus $x$ bretschneideri), and wheat (Triticum urartu).

Conclusions: This study extends the occurrence of TLEs to the plant phylum. The elements in the moss genome have amplified recently and may still be capable of transposition. The TLEs are also present in angiosperm genomes, but apparently much less abundant than in moss.
\end{abstract}

Keywords: Transposable elements, Moss, Tc1-mariner-IS630 superfamily, Tc1-like elements, Mariner-like elements, Plant genome, Evolution, Transposition activity

\section{Background}

Transposable elements (TEs) are a major component of most eukaryotic genomes. Their transposition in genomes may lead to increase in their copy numbers. TEs are classified into two categories (Class I and Class II) based on their mechanism for transposition. Class II elements are DNA transposons that adopt a 'cut-and-paste' approach catalyzed by enzymes called transposases. The elements of this class are further divided into superfamilies based on different types of transposases. All of the transposases of these elements bear a DDE/D triad motif, however, different superfamilies have distinct transposases and structural features such as the length of the duplicated target site sequences $[1,2]$. Despite the growing number of reported active TEs, the majority of transposable elements are not active $[3,4]$. These elements are important for the dynamic structure of genome during evolution $[5,6]$. The immobilized TEs can serve as raw genetic materials for genome

\footnotetext{
* Correspondence: gage.yang@utoronto.ca

'Department of Biology, University of Toronto at Mississauga, 3359

Mississauga Road, L5L 1C6 Mississauga, ON, Canada

${ }^{2}$ Cell and Systems Biology, University of Toronto, Toronto, Canada
}

tinkering [7-15]. Autonomous TEs encode and produce transposases for their mobilization. Non-autonomous elements have lost their ability to encode functional transposases and rely on other sources of transposases for transposition. An ultimate group of non-autonomous elements is miniature inverted-repeat transposable elements (MITEs). They are short elements and have high copy numbers [16-18].

Tc1-mariner-IS630 is a Class II TE superfamily first identified in nematode and insect genomes [19]. The superfamily was named after Tc1 in Caenorhabditis elegans [20], and mariner in Drosophila mauritiana [21]. This superfamily is characterized by two terminal inverted repeats (TIRs) of typically 12 to $28 \mathrm{nt}$ flanked by dinucleotide target site duplications (TSDs) of 'TA'. The transposases of this superfamily contain a triad catalytic motif consisted of two aspartic acid (D) residues and a glutamate residue (E) in Tc1-like elements (TLEs) or aspartic acid (DDD) in Mariner-like elements (MLEs) and pogo-like elements $[22,23]$. The pocket formed by these residues contains the metal ions needed in the DNA cleavage reaction during transposition [24]. Based on the number of residues 
between the second and third catalytic residues of the DDE/D motif, Tc1/mariner catalytic domains can be DD34E, DD34D, DD31-33D, DD35E, DD37D, DD37E, or DD39D, each defining a subgroup of the Tc1/mariner superfamily [18,22,25-27]. Tc1/mariner elements have been considered to be confined to animals until the recent identification of DD39D mariner-like elements and pogo-like elements in plants $[18,22,23]$. Tc1-like elements are the founding subgroup of the Tc1/mariner superfamily and they bear the DD34E triad catalytic motif [20]. Previous studies have identified TLEs in a variety of animals and fungi [23] as well as in the parasitic amoebozoa Entamoeba invadens [28]. However, to the best of our knowledge, there has been no report of TLEs outside the unikonts (animal, fungi, and amboebozoa) [29]. Previous studies have identified TLEs in a number of animal or fungal genomes, some have been demonstrated to be active, including $T c 1$ and $T c 3$ in C. elegans [20,30,31], Minos in Drosophila hydei [32], and Impala in fungus Fusarium oxysporum [33,34]. The reconstructed fish element Sleeping Beauty is also a TLE [35]. Tc1-like elements named Hydargo have been identified in Entamoeba parasites [28].

Here we report the identification of TLEs in plants. The two families of full-length TLEs in the moss (Physcomitrella patens) genome have multiple copies that contain an intact open reading frame (ORF). These ORFs are actively transcribed and presumably also translated into functional transposases in moss. TLEs were also found in the genome sequence databases of angiosperm plants.

\section{Results}

\section{Tc1-like elements in moss}

Mariner-like elements are widespread in plant genomes $[18,36]$. To investigate whether plant genomes contain TLEs, moss genome sequence databases were screened because mosses are among the first terrestrial plants. When the sequence of Tc1 transposase was used as the query sequence for BLAST search against the moss (Physcomitrella patens) genome database that has a coverage of approximately 8.6X [37], 118 high scoring hits (e-value: $<\mathrm{e}-8)$ were obtained. Close inspection of the output revealed two groups of elements that have complete terminal inverted repeats (TIRs) with terminal $5^{\prime}$-CAGT ... ACTG-3' sequences flanked by TSDs of dinucleotide 'TA'. Both groups of elements contain open reading frames for transposases bearing a DD34E motif. These characteristics suggest that these two groups are TLEs and were designated as $P p T c 1$ and $P p T c 2$. Neither of the two families has been previously described or annotated [37]. No similar elements or their transposase sequences were found in the genome of the spike moss Selaginella moellendorffii.

The full-length $P p T c 1$ elements are 1,584 bp long with TIRs of $33 \mathrm{bp}$. It has an ORF of 338 aa with two helix-turn-helix domains and a catalytic DD34E domain (Figure 1). A total of 85 copies were retrieved from the $P$. patens genome sequence database. Among them, 75 were full length bearing the intact ends with average sequence identity of $96.3 \%$, and 52 of which were highly similar copies with $>98 \%$ sequence identity, but there were no identical copies. Nine copies were found to carry an intact full-length ORF (338 aa). To gain insights into the insertion sites of PpTc1 elements, it is important to inspect the sequences homologous to the flanking sequences of $P p T c 1$ insertion sites. Such sequences that do not bear the TE insertions are called related empty sites (RESs). The sequence signatures of the TE insertion sites on RESs may reflect historical transposition events. Among the 75 full length copies, RESs can be found for the flanking sequences of 42 copies with 14 of them in AT rich simple repeat flanking sequences (Additional file 1: Figure S1). Most of the 28 RESs that are not ATrich simple repeats correspond to the sequences before insertion of elements, some (for example, that of scaffold 54) may have resulted from excision events and subsequent repairing.

The full-length $P p T c 2$ elements are 1,709 bp long, with TIRs of $33 \mathrm{bp}$ (Figure 1). A total of 22 copies of PpTc2 were retrieved from the genome database. The 20 fulllength copies have an average sequence identity of 96.6\%. PpTc2 has eight copies bearing a full-length intact 338aa ORF. Among the 20 full-length copies, RESs can be found for the flanking sequences of three copies (Additional file 1: Figure S1). While the RES of scaffold 10 clearly represents a site before insertion of an element, that of scaffold 136 may have resulted from excision events and subsequent repairing of the excision sites. Interestingly, insertion of the PpTc2 in scaffold 281 is accompanied by a duplication of a microsatellite unit at the insertion site. These RESs of $P p T c$ insertions sites demonstrated the genomic changes caused by the activity of these elements during evolution.

\section{Comparison of PpTc1 and PpTc2}

The history of activities of these elements in the genome is an important part of the evolution of these elements. According to the molecular clock theory, the mutations accumulated in each copy of an element in a TE family can be used to infer the time of divergence from their ancestral element [38]. The sequences of the ancestral element of a TE family may be approximated to the consensus sequences of the TE family. Therefore, the elements produced at the same time frame can be expected to have similar levels of sequence divergence from the ancestral element. Based on the consensus sequences of $P p T c 1$ and $P p T c 2$, the average sequence divergence score was calculated for each copy and the number of elements in a certain range of sequence divergence value 


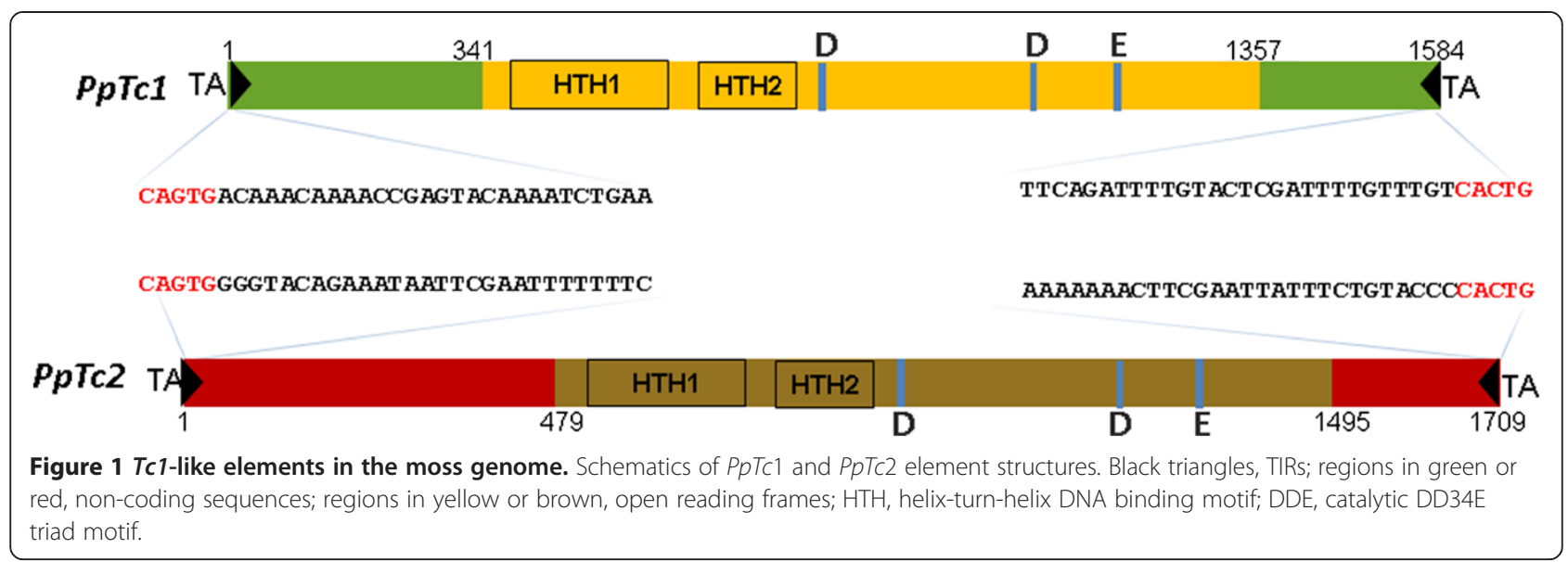

was plotted against the sequence divergence range. The $P p T c 1$ family has an average divergence value of $2.18 \pm$ $0.08 \%$ with a significant peak at $1.5 \%$ sequence divergence (Figure 2), suggesting a recent burst of amplification events of this family occurred about 1.5 million years ago and the rate of amplification has since decreased according to a rate of $1 \%$ sequence divergence per million years. The $P p T c 2$ family have an average sequence divergence value of $2.17 \pm 0.20 \%$ with the most recent peak at about $1 \%$, suggesting that $P p T c 2$, similar to $P p T c 1$, recently amplified about 1 million years ago. Interestingly, the $P p T c 2$ dynamics is similar to the cycles of TE amplification described previously [39].

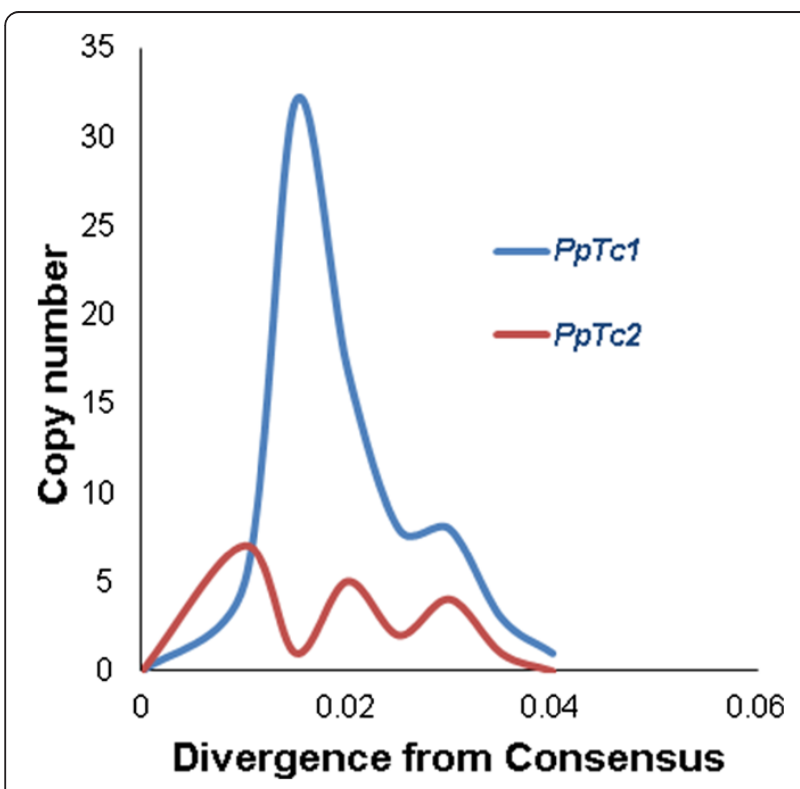

Figure 2 Sequence divergence of full-length elements of PpTc1 and PpTc2. Y-axis, number of elements; $x$-axis, level of sequence divergence from the consensus sequence of $P p T c 1$ or PpTc2 family.
Although $P p T c 1$ and $P p T c 2$ bear identical extreme terminal sequences 'CAGT' (Figure 1), their internal regions do not bear detectable DNA sequence similarities. Even the transposase coding sequences do not share significant sequence similarities between the two elements. When the putative peptide sequences of the two transposases were aligned, they share $26 \%(89 / 338)$ sequence identify with $47 \%$ positive (161/338) (Figure 3A). These results suggest that the two elements shared a very distant common ancestor. However, the very similar intra-family sequence divergence levels of the two families suggest that they may have invaded and amplified in the moss genome at a similar time during evolution.

Since the crystal structures of Mos 1 and the DNA binding domain of $T c 3$ were determined, the transposase structures of $P p T c 1$ and $P p T c 2$ can be predicted based on these templates $[24,40]$. Using Phyre2 web server, the transposase structure of Mos 1 was used by the algorithm to model the transposases. The homologous models have $100 \%$ confidence with about $95 \%$ coverage of the query sequences, suggesting highly similar protein structures between these two proteins and to the Mos 1 transposase (Figure 3B). Based on the structural features of Mos1, similar features were predicted on the models of $P p T c 1$ and $P p T c 2$ transposases. These models provide important starting information to understand the functionality of these transposases and their structural and functional deviations from other transposases in the Tc1/mariner superfamily.

\section{Expression of PpTc1 in moss}

The high intra-family sequence similarity in $P p T c 1$ and $P p T c 2$ and the presence of multiple copies of elements that contain intact transposase coding sequences indicate that they are potentially active. Expression of transposase is required for transposition activity, therefore it is important to determine whether $P p T c 1$ and $P p T c 2$ are 


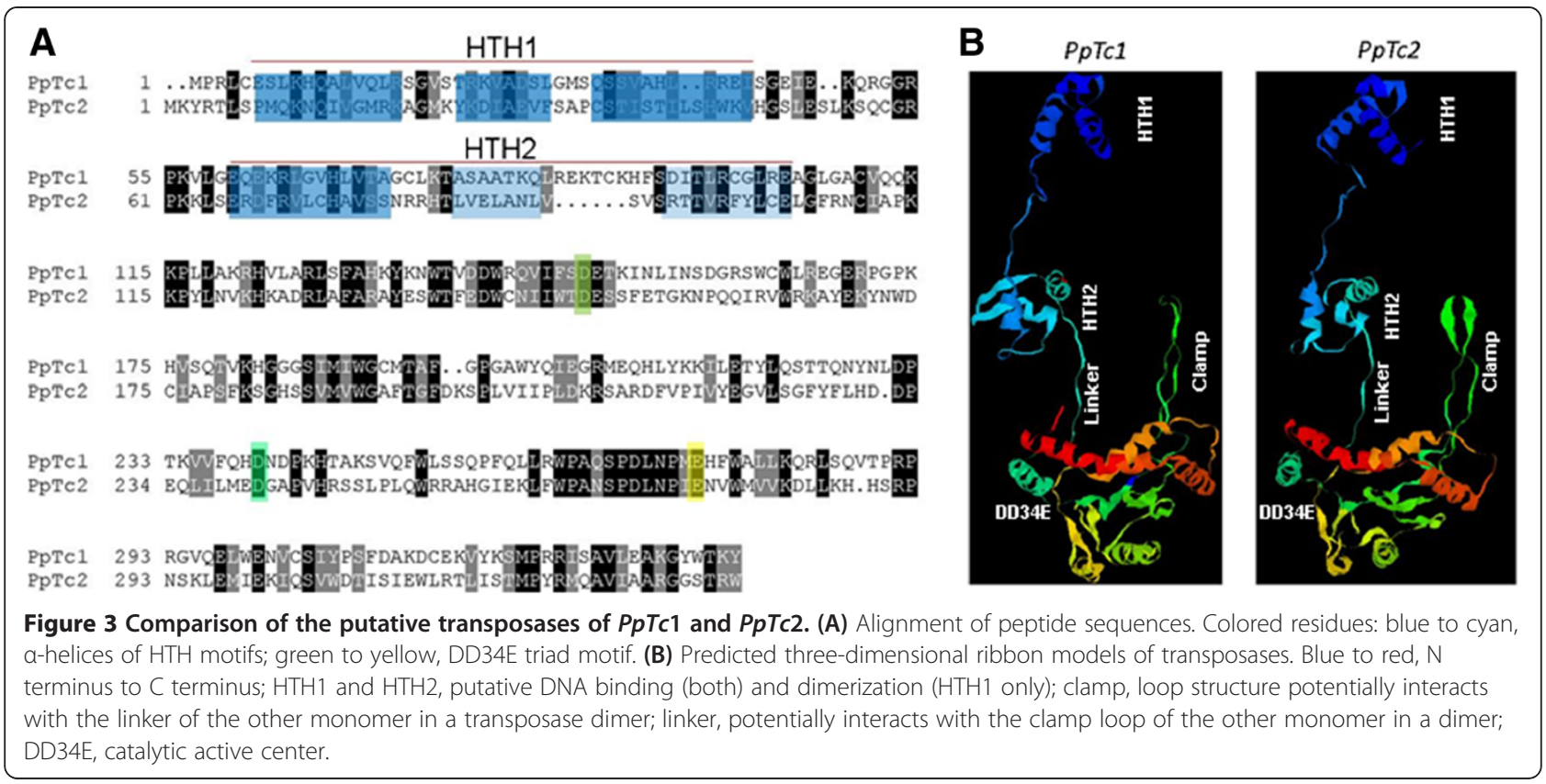

actively transcribed. Extensive sequencing of the moss transcriptome has been previously performed and reported [41]. The expressed sequence tags derived from protonemal tissue and gametophores have been analyzed extensively and resulted in an assembled transcript database Pp0409 that contains 47,557 entries (www.cosmoss.org). Expressed sequence tag coverage of the genome assembly is $98 \%$ [37]. PpTc elements and the CDS of moss actin1 gene (PpAct1) were used to retrieve assembled transcripts from the database. Compared to the 17 transcripts from PpAct1, 68 assembled transcripts containing the nucleotide sequences of the ORF region were retrieved for $P p T c 1$ and no transcript for $P p T c 2$, suggesting that the level of transcripts of PpTc1 in moss cells is higher than the constitutive gene actin 1 . Each of these transcripts corresponds to a specific copy of $P p T c 1$ element. Nine of the $P p T c 1$ transcripts can be conceptually translated into a full-length intact transposase (Figure 4, Additional file 1: Table S1). Each of these transcripts bearing intact ORFs is derived from a specific copy of the nine genomic copies of $P p T c 1$ bearing intact transposase coding sequences, suggesting that these elements are actively transcribed and yielded mature mRNA. The fact that no identical copies of $P p T c 1$ were present in the genomic sequence database suggests an attenuated transposition activity after the peak amplification of the family around 1.5 million years ago. Since TE transcripts can be degraded by siRNA and their translation may be blocked by microRNAs, the $P p T c 1$ transcripts were used to search against the small RNA databases [42-45]. However, no small RNA matching the coding sequences of $P p T c 1$ transposase gene were retrieved, suggesting that the $P p T c 1$ mRNAs are not degraded or their translation blocked, therefore may be translated into transposase proteins. Because of the abundance of the transcripts of the transposase gene, it is possible that a post-translational mechanism such as over production inhibition demonstrated for animal Tc1/mariner elements may have led to the repression of its transposition $[46,47]$. When $P p T c 2$ sequences were used to search against the assembled transcript database, no transcripts were retrieved. This suggests that the expression of the transposase genes of this family is probably repressed at the transcriptional levels.

\section{Evolutionary relationship of transposases encoded by moss TLEs to those of animal and fungal TLEs}

Since TLEs have been previously described only in animal and fungal genomes, the relationship of the moss TLEs to other TLEs will help to understand the propagation of TLEs in plant genomes. Even though there are only a few well characterized TLEs in literature, recent progress in whole genome sequencing produced TLE sequences in many different genomes. Using well characterized TLE transposase sequences including Tc1 (X01005), Tc3 (P34257.1), Minos (CAP09075.1), and Impala (AF282722), together with $P p T c 1$ and $P p T c 2$, we retrieved representative TLE sequences in different genomes from the nonredundant protein database of Genbank. The majority of these sequences were not classified therefore named as hypothetical proteins or unknown proteins. Notably, the TLE element in Rhizopus delemar was found to have at least 60 copies. After removal of redundancy of sequences belonging to the same family, together with $P p T c$ elements, the sequences were aligned with the previously 

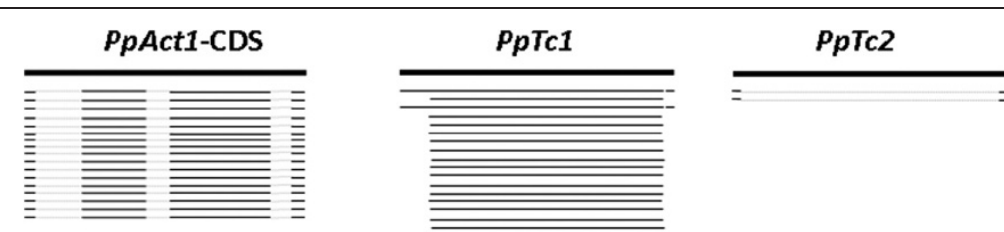

Figure 4 Transcripts from PpTc elements. Thick lines on top, query sequences; solid thin lines, matched regions between the queries and hits in the transcript database; dotted lines, unmatched regions reflecting intronic regions; the coding DNA sequence (CDS) of moss actin1 gene was used as a control.

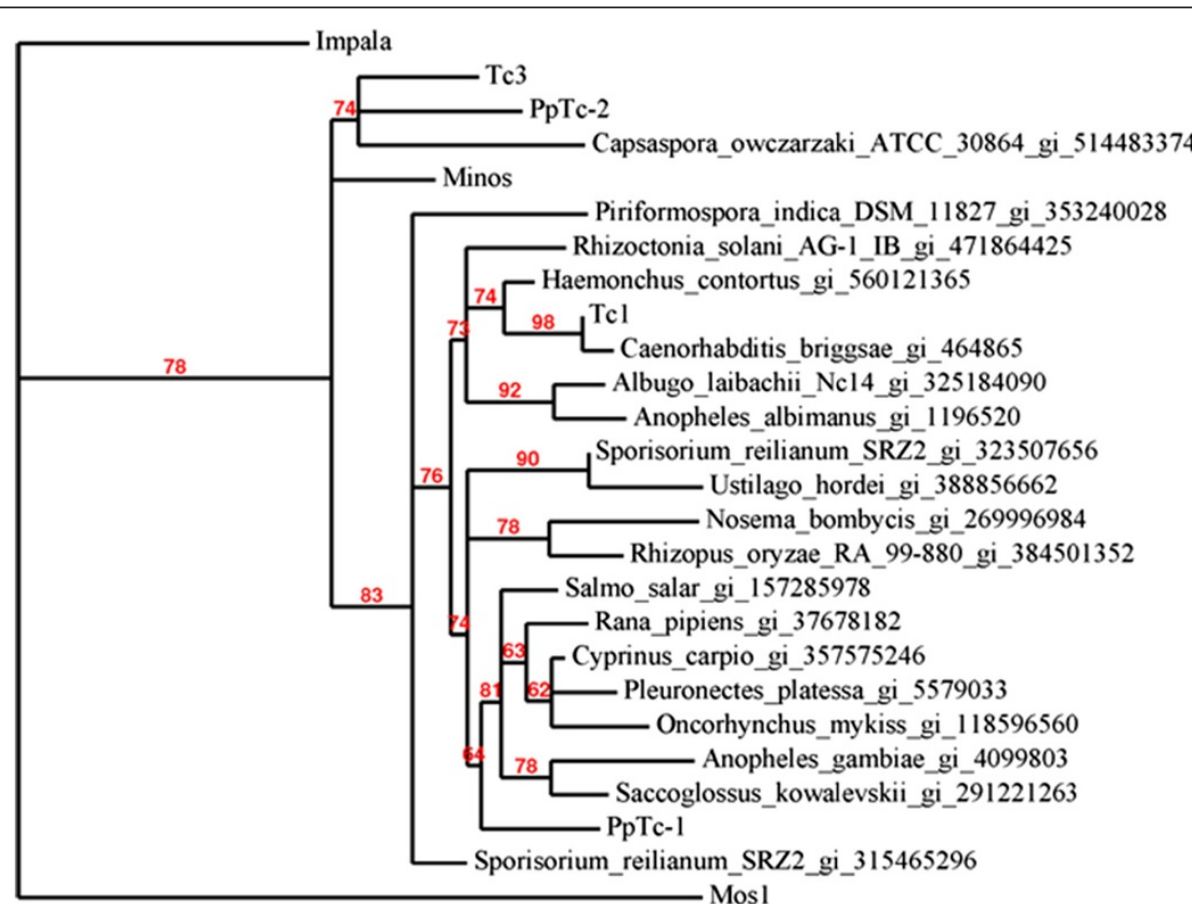

1.

Figure 5 Phylogenetic relationship of transpoases of moss TLEs to those of animal and fungal TLEs. Names, species followed by Gl numbers of each sequence; numbers on branches, percentage of bootstrap value of 1,000 reiterations. 
described TLEs and a phylogenetic tree was constructed (Figure 5). Similar to that reported previously, the branches on the phylogenetic tree of these elements have relatively low bootstrap values ( $98 \%$ to $62 \%$ ) [48]. Nevertheless, the topology of the previously analyzed elements such as Tc1, Tc3, Impala, and Minos is consistent with that shown in the previous report. Impala appeared to have branched off early from the rest of the TLEs. The rest of elements are grouped into two clades: $T c 1$ clade and $T c 3$ clade. The majority of these elements belong to the $T c 1$ clade. The fact that the phylogenetic relationship among these elements is clearly incongruent with that of their host species may suggest ancestral polymorphism or long branch attraction [49], alternatively horizontal transfer of these elements among eukaryotic species may have also contributed to the observation $[50,51]$. The two moss elements belong to different clades with $P p T c 1$ in the $T c 1$ and $P p T c 2$ in the $T c 3$ clade, further suggesting that these two elements may have different origins.

\section{TLEs in angiosperm genome sequence databases}

To determine whether TLEs have proliferated throughout plant genomes, the predicted transposase sequences of $P p T c 1$ and $P p T c 2$ were used as query sequences to search against all other plant genomic sequences in the GenBank
WGS and NR/NT databases using TBLASTN. Segments of $T c 1$-like transposase coding sequences were identified in nine angiosperm genomes including rice (Oryza sativa), dwarf birch (Betula nana), cabbage (Brassica rapa), hemp (Cannabis sativa), barley (Hordium valgare), lettuce (Lactuta sativa), poplar (Populus Trichocarpa), pear (Pyrus $x$ bretschneideri), and wheat (Triticum urartu) (Table 1). The conserved regions including at least the second (aspartic acid) and the third (glutamic acid) residues of the DD34E catalytic motif were retrieved. Most of these elements are single copies and they are not uniform in size. While TLE in the database of Oryza sativa is a complete element with intact terminal sequences, the majority of the plant TLEs are fragmented and do not encode a complete transposase. When the regions between the second D and the E residues of the DD34E motifs were aligned, conserved motifs surrounding these two residues were revealed (Figure 6A and Additional file 1: Figure S2). The conserved motifs surrounding the $\mathrm{E}$ residues of these TLEs are apparently different from those surrounding the corresponding $\mathrm{D}$ residue of the MLEs such as Mos1 (X78906), Soymar1 (AF078934.1), and Osmar5 (ACV32571.1). Among the sequenced plant genomes, the distribution of the species containing TLEs is apparently patchy (Figure 7). These results suggest that TLEs are also

Table 1 Plant Tc1-like transposases described in this study

\begin{tabular}{|c|c|c|c|c|c|}
\hline Element & Organism & Accession & ORF start & ORF end & Complete DD34E triad? \\
\hline Plant & & & & & Y: yes; N, no \\
\hline PpTc1 & Physcomitrella patens & ABEU01007491 & 7,186 & 8,199 & Y \\
\hline PpTc2 & Physcomitrella patens & ABEU01006878 & 162,826 & 161,813 & Y \\
\hline OsTc1 & Oryza sativa Indica & AAAA02041396 & 3,821 & 2,697 & Y \\
\hline BnTc1 & Betula nana & CAOK01056615 & 1,484 & 1,978 & N \\
\hline BnTc2 & Betula nana & CAOK01550459 & 168 & 1,214 & Y \\
\hline $\mathrm{BnTC3}$ & Betula nana & CAOK01014729 & 14,272 & 14,472 & N \\
\hline BnTc4 & Betula nana & CAOK01486111 & 2 & 244 & $N$ \\
\hline BrTc1 & Brassica rapa & AENI01020305 & 162 & 572 & $N$ \\
\hline BrTc2 & Brassica rapa & AENI01036930 & 17 & 328 & N \\
\hline CsTc1 & Cannabis sativa & AGQN01308320 & 302 & 517 & $N$ \\
\hline HVTc1 & Hordium valgare & CAJV010227559 & 1 & 1,684 & Y \\
\hline HvTc2 & Hordium valgare & CAJV010272453 & 49 & 555 & Y \\
\hline HvTc3 & Hordium valgare & CAJV012609061 & 1,716 & 2,114 & N \\
\hline HvTc4 & Hordium valgare & CAJV011622646 & 1 & 222 & $N$ \\
\hline LsTc1 & Lactuta sativa & AFSA01593962 & 2 & 394 & N \\
\hline LsTc2 & Lactuta sativa & AFSA01593962 & 87 & 485 & $N$ \\
\hline PtTc1 & Populus trichocarpa & AARH01030986 & 1 & 714 & Y \\
\hline PxbTc1 & Pyrus $x$ bretschneideri & AJSU01007483 & 3,055 & 3,606 & Y \\
\hline PxbTc2 & Pyrus $x$ bretschneideri & AJSU01007483 & 3,055 & 3,606 & $N$ \\
\hline TuTc1 & Triticum urartu & AOTI010070343 & 376 & 1,368 & Y \\
\hline
\end{tabular}

All TLE elements bear the D34E of the DD34E catalytic motif. 


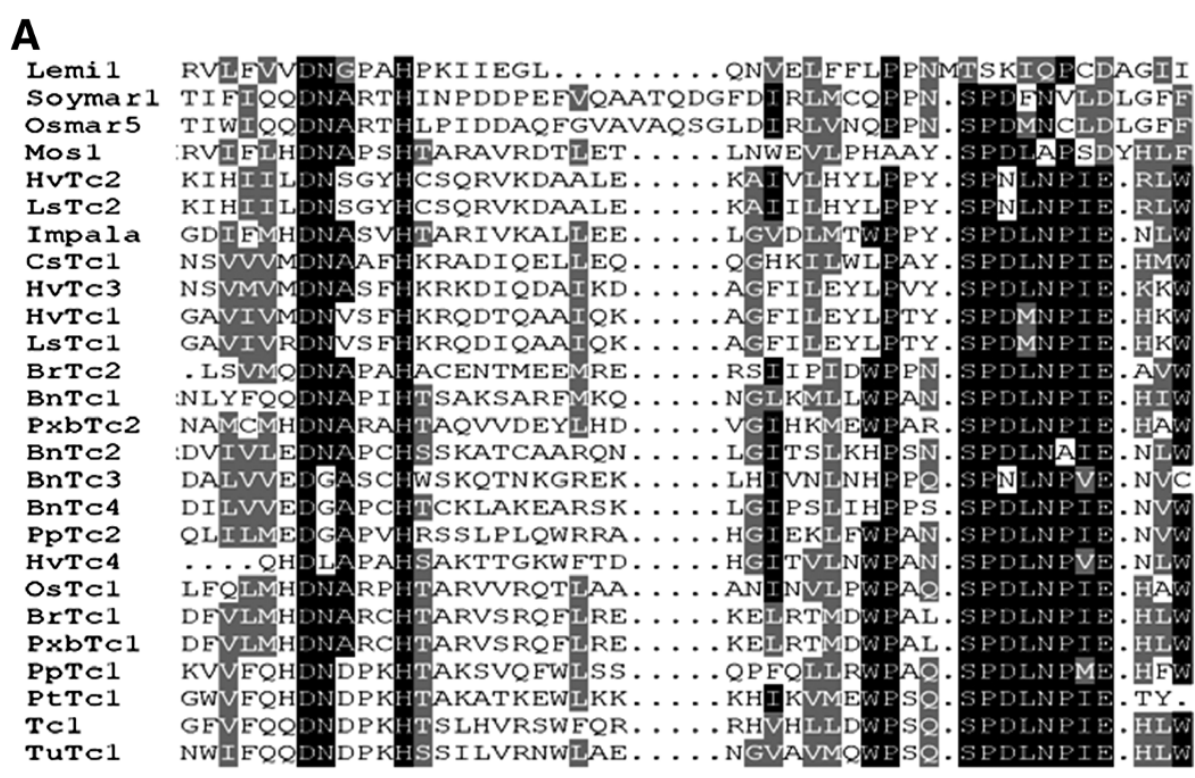

B
T C 1
P P T C 1
P P T 22
O S T C 1
H $\vee$ T C 1 *
H $\vee$ T $\subset 4$
$\mathrm{P} \times \mathrm{b}$ T $\mathrm{C}-1$
P xb T c 2 *
P t T c 1 *
T $\mathrm{T}$ T $\mathrm{C}-1$

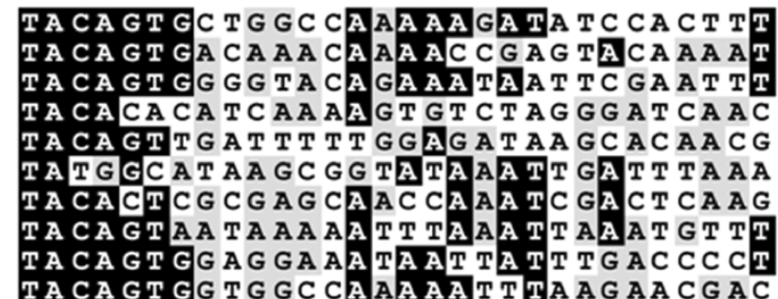

Figure 6 Sequence alignment of the catalytic motifs of transposases (A) and end sequences (B) of plant TLEs. (A) The regions containing the DDE/D catalytic motifs of the transposase sequences. Plant MLEs are shown at the bottom. (B) The terminal sequences of plant TLEs and TC1. The degree of background shading indicates different levels of conservation of sequences. Asterisks indicate elements that only have one end present in a genomic contig. Abbreviation for species names: Os, Oryza sativa; Bn, Betula nana; Br, Brassica rapa); Cs, Cannabis sativa; Hv, Hordium valgare; Ls, Lactuta sativa; Pt, Populus trichocarpa; Pb, Pyrus x bretschneideri; Tu, Triticum urartu.

present in angiosperm genomes, but are much less abundant than in the moss genome.

\section{Discussion}

The identification of TLEs in plant genomes expanded our knowledge on the distribution and diversity of Tc1/ mariner elements. Elements belonging to the marinersubgroup have been found to be widespread in plant genomes [18]. TLEs, however, have not been previously reported in plants. In fact, $P p T c 1$ and $P p T c 2$ are the first Tc1/mariner elements described in moss. They not only expand the range of distribution of TLEs into plants, but also provide information for the development of TEbased tools for gene discovery in moss in the future.

$P p T c$ elements have undergone a recent wave of proliferation. The results suggest that their transposition activities appear to have subsequently been contained in the current moss genome. Although most copies of $P p T c$ elements have lost the capacity to encode a functional transposase due to mutations that interrupt the transposase coding sequences, both families have members bearing full length intact transposase-coding genes and $P p T c 1$ elements are actively expressed in moss. These observations indicate that, even though the transposition activity of $P p T c 1$ may have been attenuated, it may still be modestly active. In addition, since the genome was sequenced with shot gun approaches, the reads for these repetitive sequences may have been misassembled. Therefore, it is possible that identical $P p T c 1$ sequences are present in the genome. The absence of transcripts from $P p T c 2$ may indicate a high level of repression of transposition. It remains mysterious how these elements are repressed. It is possible that, under certain environmental factors, these elements may become fully active in transposition. Alternatively, the activities of these elements may be restricted to certain tissues/organs or specific temporal stages during the life cycle of the plant. Further investigation on the repression of the transposition activities of both families will facilitate our understanding of the interaction between TEs and their host genomes. 


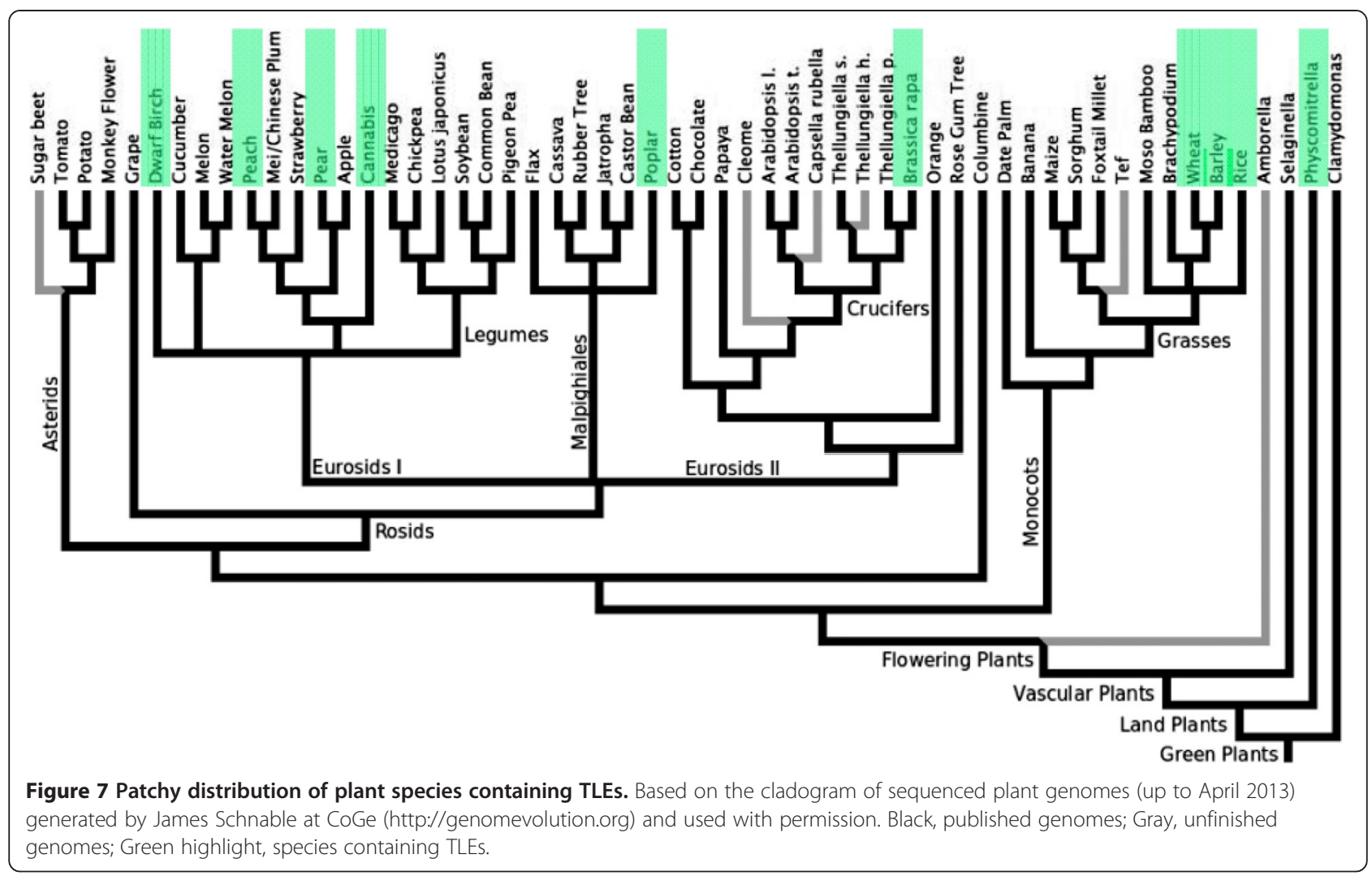

\section{Conclusions}

TLEs are present in plant genomes. The two families of TLEs in the moss genome have recently amplified 1 to 2 million years ago. These families contain elements that are potentially capable of transposition but their transposition activities appear to have been attenuated. TLEs were also identified in the genome databases of angiosperm plants, suggesting their distribution in multiple plant orders. The results presented in this report further our understanding of the evolutionary history of Tc1/mariner elements and provide important information for future investigations into the interaction between TEs and host genomes.

\section{Methods}

\section{Retrieval of moss Tc1-like elements}

To identify transposons related to $T c 1$-like elements, the $T c 1$ transposase peptide sequence was used as the query sequence to search against GenBank databases of $P$. patens genome with the default parameters. Each returned hit was retrieved and inspected for TIRs. Complete elements were searched against its host genome to obtain the members of its family. Nucleotide sequences of full-length TLE copies were retrieved with MITE Analysis Kit function MEMBER (http://labs.csb.utoronto.ca/yang/MAK/) [52,53]. Members of each family were retrieved with MAK with zero tolerance for end mismatches.

\section{Characterization of moss TLEs}

Alignments of all retrieved members in each $P p T c$ family were obtained with CLUSTAL, and a consensus sequence was generated. The elements were conceptually translated and scanned for long ORFs with the APE program (http:// biologylabs.utah.edu/jorgensen/wayned/ape/). HTH motifs were predicted with NPS webserver (http://npsa-pbil.ibcp. fr/cgi-bin/npsa_automat.pl?page=/NPSA/npsa_hth.html) and the conserved domain database at NCBI. The putative models of $P p T c 1$ and $P p T c 2$ were predicted with Phyre2 (http://www.sbg.bio.ic.ac.uk/phyre2/). Sequence alignments were performed with MUSCLE at the EBI webserver (http://www.ebi.ac.uk/Tools/msa/muscle/) and the phylogenetic tree was constructed with Phylogy.fr (www.phylogeny.fr) with 1,000 bootstrap reiterations.

\section{Sequence divergence of PpTc1 and PpTc2 families}

To calculate the average sequence divergence of a family, the consensus sequence of each family was constructed. The consensus sequence was used as the input for the Divergence function of MAK. Each divergence value is the complementary percentage of the similarity value in the pairwise alignment of a copy and the consensus sequence. The output contains the sequence divergence values for each member. The average divergence for each family was calculated. To plot the number of elements against 
divergence, values of individual divergence were grouped into bins of $0.5 \%$ and the number of elements in each bin was counted. The overall sequence similarity for a family is calculated as the complement of the average sequence divergence.

\section{Expression analysis of $P p T c$ families}

Moss TLEs PpTc1 and PpTc2 (ABEU01007491 and ABEU01006878, respectively) were used to search against the assembled transcripts database Pp0409 on the moss genome browser (http://www.cosmoss.org/) [41]. Returned hits were inspected for a long ORF that encodes a transposase bearing a DD34E catalytic motif. The loci of transcripts were cross-referenced to the nucleotide BLAST hits to remove redundancy. The sequences were also used to search for moss small RNA databases [42-45].

\section{Analyses of TLEs in other plant genome databases} Plant genome databases WGS and NR/NT were searched at NCBI using TBLASTN with the peptide sequences of the putative transposases of $P p T c 1$ and $P p T c 2$. Hits and their flanking sequences were retrieved to identify putative transposase or TIR sequences.

\section{Additional file}

Additional file 1: Table $\mathbf{S 1}$. Transcripts of $P p T C 1$ that produce a conceptual full-length DD34E transposase. Figure S1. Related empty sites (RESs) for moss TLEs. Figure S2. Sequence alignment of plant TLEs and other Tc1/mariner representative elements using all predicted peptide sequences.

\section{Abbreviations}

MLE: Mariner-like element; TE: Transposable element; TIR: Terminal inverted repeat; TLE: TC1-like element; TSD: Target site duplication.

\section{Competing interests}

The author(s) declare that they have no competing interests.

\section{Authors' contributions}

GY conceived the study. YL and GY designed and performed the analyses. $Y L$ and $G Y$ drafted the manuscript. $Y L$ and GY revised the manuscript. GY edited and finished the manuscript. Both authors read and approved the final manuscript.

\section{Acknowledgements \\ We would like to thank Dr. Isam Fattash for assistance on the analyses of the expression of moss elements. Funded by the Natural Sciences and Engineering Research Council (NSERC) Discovery Grant (Canada) (RGPIN 371565 to GY, RGPIN-2014-04709), Canadian Foundation for Innovation (CFI24456 and IOF-12 to GY), Ontario Research Foundation (ORF24456 to GY), and the University of Toronto. The funding sources played no role in research design; the collection, analysis, and interpretation of data; the writing of the manuscript; or in the decision to submit the manuscript for publication.}

Received: 7 March 2014 Accepted: 12 May 2014

Published: 3 June 2014

\section{References}

1. Yuan YW, Wessler SR: The catalytic domain of all eukaryotic cut-and-paste transposase superfamilies. Proc Natl Acad Sci U S A 2011, 108:7884-7889.

2. Wicker T, Sabot F, Hua-Van A, Bennetzen JL, Capy P, Chalhoub B, Flavell A, Leroy $P$, Morgante M, Panaud O, Paux E, SanMiguel P, Schulman AH: A unified classification system for eukaryotic transposable elements. Nat Rev Genet 2007, 8:973-982.

3. Huang CR, Burns KH, Boeke JD: Active transposition in genomes. Annu Rev Genet 2012, 46:651-675.

4. Feschotte C, Pritham EJ: DNA transposons and the evolution of eukaryotic genomes. Annu Rev Genet 2007, 41:331-368.

5. Yang LX, Bennetzen $\mathrm{J}$ : Distribution, diversity, evolution, and survival of Helitrons in the maize genome. Proc Natl Acad Sci U S A 2009, 106:19922-19927.

6. Du C, Fefelova N, Caronna J, He LM, Dooner HK: The polychromatic Helitron landscape of the maize genome. Proc Natl Acad Sci U S A 2009, 106:19916-19921

7. Rebollo R, Romanish MT, Mager DL: Transposable elements: an abundant and natural source of regulatory sequences for host genes. Annu Rev Genet 2012, 46:21-42.

8. Jordan IK: Evolutionary tinkering with transposable elements. Proc Natl Acad Sci U S A 2006, 103:7941-7942.

9. Cowley M, Oakey RJ: Transposable elements re-wire and fine-tune the transcriptome. PLoS Genet 2013, 9:e1003234

10. Mukamel Z, Tanay A: Hypomethylation marks enhancers within transposable elements. Nat Genet 2013, 45:717-718.

11. Feschotte $\mathrm{C}$ : Transposable elements and the evolution of regulatory networks. Nat Rev Genet 2008, 9:397-405.

12. Kraitshtein Z, Yaakov B, Khasdan V, Kashkush K: Genetic and epigenetic dynamics of a retrotransposon after allopolyploidization of wheat. Genetics 2010, 186:801-812.

13. Jiang N, Ferguson AA, Slotkin RK, Lisch D: Pack-Mutator-like transposable elements (Pack-MULEs) induce directional modification of genes through biased insertion and DNA acquisition. Proc Natl Acad Sci U S A 2011, 108:1537-1542

14. Naito K, Zhang F, Tsukiyama T, Saito H, Hancock CN, Richardson AO, Okumoto Y, Tanisaka T, Wessler SR: Unexpected consequences of a sudden and massive transposon amplification on rice gene expression. Nature 2009, 461:1130-U1232.

15. Hollister JD, Smith LM, Guo YL, Ott F, Weigel D, Gaut BS: Transposable elements and small RNAs contribute to gene expression divergence between Arabidopsis thaliana and Arabidopsis lyrata. Proc Natl Acad Sci U S A 2011, 108:2322-2327.

16. Fattash I, Rooke R, Wong A, Hui C, Luu T, Bhardwaj P, Yang G: Miniature Inverted-repeat Transposable Elements (MITEs): discovery, distribution and activity. Genome 2013, 56:475-486.

17. Jiang $N$, Feschotte $C$, Zhang $X Y$, Wessler SR: Using rice to understand the origin and amplification of miniature inverted repeat transposable elements (MITEs). Curr Opin Plant Biol 2004, 7:115-119.

18. Feschotte C, Wessler SR: Mariner-like transposases are widespread and diverse in flowering plants. Proc Natl Acad Sci U S A 2002, 99:280-285.

19. Plasterk RH, Izsvak Z, Ivics Z: Resident aliens: the Tc1/mariner superfamily of transposable elements. Trends Genet 1999, 15:326-332.

20. Eide D, Anderson P: Transposition of Tc1 in the Nematode Caenorhabditis-Elegans. Proc Natl Acad Sci U S A 1985, 82:1756-1760.

21. Jacobson JW, Medhora MM, Hartl DL: Molecular structure of a somatically unstable transposable element in Drosophila. Proc Natl Acad Sci U S A $1986,83: 8684-8688$

22. Feschotte $C$, Mouches $C$ : Evidence that a family of miniature invertedrepeat transposable elements (MITEs) from the Arabidopsis thaliana genome has arisen from a pogo-like DNA transposon. Mol Biol Evol 2000, 17:730-737.

23. Robertson HM: Evolution of DNA transposons in eukaryotes. In Mobile DNA II. Edited by Craig NL, Craigie R, Gellert M, Lambowitz AM. Washington, DC: ASM Press; 2002:1093-1110.

24. Richardson JM, Colloms SD, Finnegan DJ, Walkinshaw MD: Molecular architecture of the Mos1 paired-end complex: the structural basis of DNA transposition in a eukaryote. Cell 2009, 138:1096-1108.

25. Doak TG, Doerder FP, Jahn CL, Herrick G: A proposed superfamily of transposase genes - transposon-like elements in ciliated protozoa and a common D35e motif. Proc Natl Acad Sci U S A 1994, 91:942-946. 
26. Shao HG, Tu ZJ: Expanding the diversity of the IS630-Tc1-mariner superfamily: discovery of a unique DD37E transposon and reclassification of the DD37D and DD39D transposons. Genetics 2001, 159:1103-1115.

27. Negoua A, Rouault JD, Chakir M, Capy P: Internal deletions of transposable elements: the case of Lemi elements. Genetica 2013, 141:369-379.

28. Pritham EJ, Feschotte C, Wessler SR: Unexpected diversity and differential success of DNA transposons in four species of Entamoeba protozoans. Mol Biol Evol 2005, 22:1751-1763.

29. Keeling PJ, Burger G, Durnford DG, Lang BF, Lee RW, Pearlman RE, Roger AJ, Gray MW: The tree of eukaryotes. Trends Ecol Evol 2005, 20:670-676

30. Collins J, Forbes E, Anderson P: The Tc3 family of transposable genetic elements in Caenorhabditis-elegans. Genetics 1989, 121:47-55.

31. Ruan KS, Emmons SW: Precise and imprecise somatic excision of the transposon Tc1 in the nematode C-elegans. Nucleic Acids Res 1987, 15:6875-6881.

32. Pavlopoulos A, Oehler S, Kapetanaki MG, Savakis C: The DNA transposon Minos as a tool for transgenesis and functional genomic analysis in vertebrates and invertebrates. Genome Biol 2007, Suppl 1:S2

33. Carr PD, Tuckwell D, Hey PM, Simon L, D'Enfert C, Birch M, Oliver JD, Bromley MJ: The transposon impala is activated by low temperatures: use of a controlled transposition system to identify genes critical for viability of Aspergillus fumigatus. Eukaryot Cell 2010, 9:438-448.

34. Hua-Van A, Langin T, Daboussi MJ: Aberrant transposition of a Tc1-mariner element, impala, in the fungus Fusarium oxysporum. Mol Genet Genomics 2002, 267:79-87.

35. Ivics Z, Hackett PB, Plasterk RH, Izsvak Z: Molecular reconstruction of Sleeping beauty, a Tc1-like transposon from fish, and its transposition in human cells. Cell 1997, 91:501-510.

36. Jarvik T, Lark KG: Characterization of soymar1, a mariner element in soybean. Genetics 1998, 149:1569-1574.

37. Rensing SA, Lang D, Zimmer AD, Terry A, Salamov A, Shapiro H, Nishiyama T, Perroud PF, Lindquist EA, Kamisugi Y, Tanahashi T, Sakakibara K, Fujita T, Oishi K, Shin-I T, Kuroki Y, Toyoda A, Suzuki Y, Hashimoto S, Yamaguchi K, Sugano S, Kohara Y, Fujiyama A, Anterola A, Aoki S, Ashton N, Barbazu WB, Barker E, Bennetzen JL, Blankenship R, et al: The Physcomitrella genome reveals evolutionary insights into the conquest of land by plants. Science 2008, 319:64-69.

38. Kapitonov V, Jurka J: The age of Alu subfamilies. J Mol Evol 1996, 42:59-65.

39. Le Rouzic A, Boutin TS, Capy P: Long-term evolution of transposable elements. Proc Natl Acad Sci U S A 2007, 104:19375-19380.

40. van Pouderoyen G, Ketting RF, Perrakis A, Plasterk RHA, Sixma TK: Crystal structure of the specific DNA-binding domain of Tc3 transposase of C-elegans in complex with transposon DNA. EMBO J 1997, 16:6044-6054

41. Lang D, Eisinger J, Reski R, Rensing SA: Representation and high-quality annotation of the Physcomitrella patens transcriptome demonstrates a high proportion of proteins involved in metabolism in mosses. Plant Biol (Stuttg) 2005, 7:238-250.

42. Griffiths-Jones S, Saini HK, Van Dongen S, Enright AJ: miRBase: tools for microRNA genomics. Nucleic Acids Res 2008, 36:D154-D158.

43. Fattash I, Voss B, Reski R, Hess WR, Frank W: Evidence for the rapid expansion of microRNA-mediated regulation in early land plant evolution. BMC Plant Biol 2007, 7:13

44. Talmor-Neiman M, Stav R, Klipcan L, Buxdorf K, Baulcombe DC, Arazi T: Identification of trans-acting siRNAs in moss and an RNA-dependent RNA polymerase required for their biogenesis. Plant J 2006, 48:511-521.

45. Axtell MJ, Jan C, Rajagopalan R, Bartel DP: A two-hit trigger for siRNA biogenesis in plants. Cell 2006, 127:565-577.

46. Lohe AR, Hartl DL: Autoregulation of mariner transposase activity by overproduction and dominant-negative complementation. Mol Biol Evol 1996, 13:549-555.

47. Lampe DJ, Grant TE, Robertson HM: Factors affecting transposition of the Himar1 mariner transposon in vitro. Genetics 1998, 149:179-187.

48. Feschotte C, Swamy L, Wessler SR: Genome-wide analysis of mariner-like transposable elements in rice reveals complex relationships with stowaway miniature inverted repeat transposable elements (MITEs). Genetics 2003, 163:747-758.

49. Capy $P$, Anxolabehere $D$, Langin T: The strange phylogenies of transposable elements - are horizontal transfers the only explanation. Trends Genet 1994, 10:7-12
50. Biedler JK, Sha HG, Tu ZJ: Evolution and horizontal transfer of a DD37E DNA transposon in mosquitoes. Genetics 2007, 177:2553-2558.

51. Robertson HM: The mariner transposable element is widespread in insects. Nature 1993, 362:241-245.

52. Yang G, Hall TC: MAK, a computational tool kit for automated MITE analysis. Nucleic Acids Res 2003, 31:3659-3665.

53. Janicki M, Rooke R, Yang G: Bioinformatics and genomic analysis of transposable elements in eukaryotic genomes. Chromosome Res 2011, 19:787-808.

doi:10.1186/1759-8753-5-17

Cite this article as: Liu and Yang: Tc1-like transposable elements in plant genomes. Mobile DNA 2014 5:17.

\section{Submit your next manuscript to BioMed Central and take full advantage of:}

- Convenient online submission

- Thorough peer review

- No space constraints or color figure charges

- Immediate publication on acceptance

- Inclusion in PubMed, CAS, Scopus and Google Scholar

- Research which is freely available for redistribution 